\title{
On Construction of Bit-Interleaved Coded Modulation Systems with Iterative Decoding
}

\author{
Quoc Khanh Mai ${ }^{1}$, The Cuong Dinh ${ }^{1}$, Takeshi Hashimoto ${ }^{2}$ \\ ${ }^{1}$ Radio Electronics Engineering, Le Qui Don Technical University, Hanoi, Vietnam \\ ${ }^{2}$ Electronics Engineering, University of Electro-Communication, Tokyo, Japan \\ Correspondence: The Cuong Dinh, cuongdt@mta.edu.vn \\ Manuscript communication: received 20 November 2010, accepted 16 January 2011
}

\begin{abstract}
A new construction of Bit-Interleaved Coded Modulation systems with Iterative Decoding (BICM-ID) is proposed to achieve the best performance over white additive Gaussian noise (AWGN) channels, assuming that the ideal feedback (IF) holds for iterative decoding. For a class of so-called regular IF mappings, new upper bounds for error probabilities are presented for both cases of BICM-ID systems using overall and in-line interleaving. Search results for component Recursive Systematic Convolutional (RSC) codes with and without puncturing are reported for 4-PSK and 8-PSK signal sets.
\end{abstract}

Keywords- Bit-Interleaved Coded Modulation, Rate Compatible Punctured Convolutional codes.

\section{INTRODUCTION}

Geometrical uniformity together with Geometrically Uniform (GU) signal constellation, GU binary labelings and related GU codes were first introduced by Forney [1] as an intelligent generalization and good extension of earlier works of Calderbank and Sloane [2], Benedetto [3]. The linearity of the coding systems and the regularity of the signal constellation in use were combined to provide the so-called uniform error property (UEP). With UEP, analysis and design of signal space codes are simplified because only the all-zero sequence must be considered to evaluate any kind of code performance.

Geometrical uniformity was then extended to design multidimensional trellis coded modulation (TCM) codes (see $[4,5]$ and references therein). However, there were at least two problems to be solved. Firstly, it is complicated to apply the concept of GU in the analysis and design of an important class of coded modulation systems, namely, the widely interested bit-interleaved coded modulation (BICM) [6] schemes without or with iterative decoding (ID) $[7,8]$ due to the interleaving at bit level and the general mapping to the signal constellation. Secondly, for most transmission systems involving the binary information sequences that must be transmitted to the receiver, the bit error probability (BEP) is a more important performance measure than the symbol sequence error probability (SEP). To solve the second problem, Garello et al. [9] introduced the concept of bit geometrically uniform (BGU) labelings which should help to design encoders with the socalled uniform bit error property (UBEP). Though [9] reported some important theoretical results on BGU, the definition of the BGU labelings was so strict that there were only a few BGU labelings with usual con- stellations (PSK, PAM, and QAM). The application of BGU was the serially concatenated trellis codes with UBEP.

The definition of BGU labelings given in [9] was related to maximum likelihood (ML) decoding. To relax this very strong condition in order to widen the use of the UBEP concept, we note that the complex ML decoding/detection has been recently replaced by suboptimum iterative decoding/detection. At high enough SNRs, the performance of ID comes very close to the performance of ML decoding due to the ideal feedback (IF) [10].

A good overview of theoretical foundations of BICM and BICM-ID can be found in [11], together with efficient tools for the error probability analysis. Nevertheless, in research of BICM-ID systems, most effort is concentrated on the design of signal constellations and signals' labelings (see [12] and references therein) to obtain the best BER over Gaussian as well as Rayleigh channels. There are three topics for constructing a BICM-ID system:

1) how to select the RSC code for a certain signal constellation with a certain mapping rule,

2) which kind of bit interleavers (overall or in-line) can be used, and

3) which output bit (the systematic bit or parity bits) of the RSC code must be better protected.

In this paper, we associate the regular IF mappings with the UEP, assuming that the feedback in iterative decoding is perfect. Theoretical results which appeared in the form of some upper bounds on the error probabilities of both BICM-ID systems with overall and inline interleaving are used in a full search for good component binary RSC codes, to end up the argument on which kind of bits we have to better protect. 


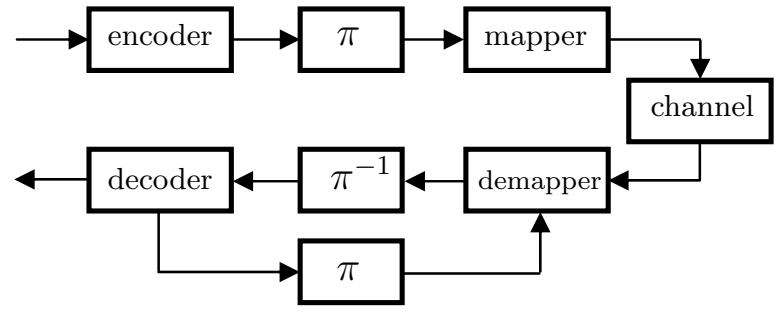

Figure 1. The system model

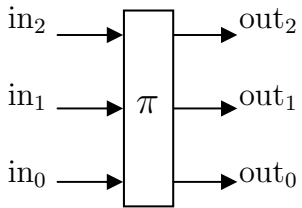

a)

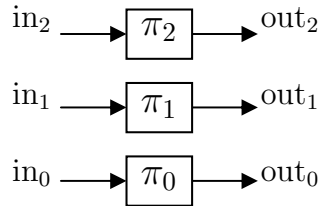

b)
Figure 2. Overall a) and in-line b) bit interleavers.

\section{SYSTEM Models}

We consider a BICM-ID system as depicted in Figure 1 with a feedback from the channel decoder to the demapper for iterative demapping and decoding. The bit interleaving has two types: the overall interleaving and the in-line interleaving (see Figure 2). Although the in-line interleavers form only a subset of overall interleavers, they have some nice properties that can be employed to improve the system performance.

A sequence of $K$ information bits is encoded by a binary RSC code whose $N=K / R$ output bits are bitinterleaved by a random interleaver $\pi$. Here $R$ is the rate of the encoder. At time index $t$, the bits $c_{t}=$ $\left(c_{t}^{1}, c_{t}^{2}, \ldots, c_{t}^{m}\right), t=1, \ldots, N / m$, from the interleaved sequence are mapped to a symbol $s_{t} \in S$ by the labeling $c_{t}=\mu\left(s_{t}\right)$ defined below.

For a finite constellation $S$ with cardinality $2^{m}$, a binary labeling $\mu[S, m]$ is a one-to-one function that associates a distinct $m$-bit label $c=\mu(s)$ to each signal $s \in S$. For $s_{i} \in S$ let $c_{i}=\left(c_{i}^{1}, c_{i}^{2}, \ldots, c_{i}^{m}\right), i=0, \ldots, 2^{m}-1$ be the binary label such that $c_{i}=\mu\left(s_{i}\right)$. Let $s_{i}$ and $s_{i^{*}}$ be a pair such that $c_{i}^{j} \oplus c_{i^{*}}^{j}=1, c_{i^{*}}=\mu\left(s_{i^{*}}\right)$. We define the $j$-th bit distance associated with a signal $s_{i} \in S$ as the Euclidean distance $d_{E}\left(s_{i}, j\right)=\left\|s_{i}-s_{i^{*}}\right\|$. Obviously, $d_{E}\left(s_{i}, j\right)=d_{E}\left(s_{i^{*}}, j\right)$. In general, $d_{E}\left(s_{i}, j\right)$ depends on the signal $s_{i}$ and there may be up to $2^{m-1}$ different $d_{E}\left(s_{i}, j\right)$ for each $j$.

Definition 1 A binary labeling $\mu[S, m]$ for a constellation $S$ is an IF-regular labeling if for each $j \in\{1, \ldots, m\}$ the bit distance does not depend on the signal, i.e., $d_{E}\left(s_{i}, j\right)$ is the same for each signal $s_{i}$, say, $d_{E}\left(s_{i}, j\right)=d_{E}^{j}$.

In this paper, for an integer $4 \leq M \leq 12$ we consider M-PSK signals $\{\exp (j 2 \pi p / M), p=0,1, \ldots, M-1\}$. For $m \leq \log _{2} M$, the $2^{m}$-point signal set $S$ is selected from the M-PSK signal set. We represent the labeling $\mu[S, m]$ of the constellation $S$ by a vector $\left(p_{0}, p_{1}, \ldots p_{2^{m}-1}\right)$ with $0 \leq p_{i} \leq M-1$ and $0 \leq i \leq$

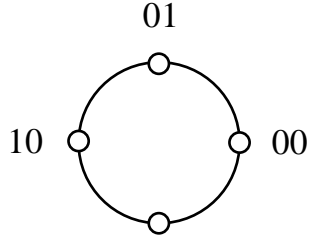

11

a)

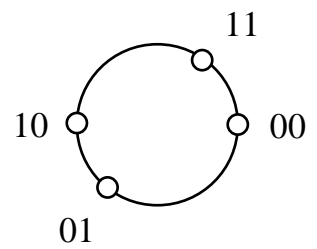

b)
Figure 3. IF-regular labelings of the 4PSK constellation

$2^{m}-1$. This means the signal point $\exp \left(j 2 \pi p_{i} / M\right)$ is associated with the label which is a binary representation of the decimal number $i$. For example, SP-A = $[0,1,2,3]$ is a set partitioning labeling of the standard 4PSK constellation (Figure 3a). SSP $=[0,4,3,1]$ is the semi-set partitioning labeling of 4 signal points taken from the 6PSK constellation (Figure 3b). These labelings are IF-regular. Clearly, if $\mu[S, m]$ is an IF-regular labeling of the constellation $S$, any length- $m$ permutation acting on $m$-bit labels gives rise to an IF-regular labeling, too. We distinguish IF-regular labelings SP-A and SP-B $=[0,2,1,3]$, which is a transformation of SP-A by permuting the first bit with the second bit in each label of signal points. While the type of mapping (SP or SSP) is determining the set of bit distances, the permutation determines the position of each RSC encoder output bit in the $m$-bit label of the signal.

We assume the ideal feedback. Consider the AWGN channel with $2^{m}$-ary modulation. Due to the bit interleaver, it can be seen as encoded bits are sent over $m$ separate parallel binary channels [7]. If the labeling is IF-regular, each of these binary channels is characterized by a bit distance. Thus, the $j$-th channel, $j \in\{1, \ldots, m\}$, has a bit distance $d_{E}^{j}$ and an equivalent bit energy $E_{b}^{j}=\left(d_{E}^{j}\right)^{2} / 4$.

In the overall bit interleaving system, each bit in the interleaver's output sequence is sent (with probability $1 / m$ ) through one of the above mentioned $m$ binary channels of energy $E_{b}^{j}=\left(d_{E}^{j}\right)^{2} / 4, j \in\{1, \ldots, m\}$. The average modulation energy then is $\left[\left(d_{E}^{1}\right)^{2}+\ldots+\right.$ $\left.\left(d_{E}^{m}\right)^{2}\right] / 4 m$. Transmission of a symbol can be then imagined as the consecutive $m$ uses of the average binary channel, each lasts $T_{C}=T_{S} / m$ seconds, where $T_{S}$ is the symbol duration. The equivalent noise bandwidth is $m$ times as large as the original transmission bandwidth. If $N_{0}$ is the noise density of the AWGN channel, then the noise density of the equivalent binary channel is $N_{0}^{*}=N_{0} / m$.

Remark 1 Under the assumption of IF and IF-regular labelings the performance of the BICM-ID system in error floor range is equivalent to the ML performance of the component RSC code over an equivalent AWGN channel with BPSK modulation of a signal-to-noise power ratio (SNR)

$$
\frac{E_{b}}{N_{0}^{*}}=\frac{1}{4 R N_{0}} \sum_{j=1}^{m}\left(d_{E}^{j}\right)^{2}
$$


For a large information bit frame $K$, we can assume without loss of generality a terminated to the all-zero state RSC code whose codewords in fact form a linear block code of rate $K / N$. Since interleaving is a linear transformation of each codeword, at the interleaver's output we have another block code.

Remark 2 The linearity of the code and the IF-regularity of the labeling of the signal constellation (by Definition 3) implies that the UEP holds for the BICM-ID in consideration.

The UEP simplifies the code performance analysis. Most of upper bounds on error probabilities of BICMID systems are development of the one described in [9], based on the serial concatenation structure of the system. In this paper, we use an approach of transfer function upper bounds of RSC codes.

\section{UpPER Bounds on Bit ERror Probabilities}

\subsection{The BICM-ID System with Overall Interleaving}

Theorem 1 Consider a BICM-ID system with overall bit interleaving and transmission of a rate $k / n$ RSC code over an AWGN channel with one-side noise power spectral density $N_{0}$. Under the assumption of IF and IF-regular labeling, the BEP of the system is upper bounded as follows

$$
P_{b}<\left.\frac{1}{k} \frac{\partial T(D, I, L)}{\partial I}\right|_{I=1, L=1},
$$

where the dummy variable $D$ is given as

$$
D=\exp \left\{-\frac{1}{4 N_{0}} \sum_{j=1}^{m}\left(d_{E}^{j}\right)^{2}\right\} .
$$

First, we note that the bound (1) belongs to a class of so called transfer function upper bounds, so we can apply to this bound all tightening techniques and numerical computation described in [12]. Second, the bound (1) differs from the classical Viterbi bound only in computation of the dummy variable $D$, more exactly, in the exponent which represents the SNR. Thus, one can approximately compute the gain of the BICM-ID system compared with performance of the RSC code over an AWGN channel with BPSK and SNR equal to

$$
\frac{E_{S}}{m R\left(N_{0} / m\right)}=\frac{E_{S}}{R N_{0}}=\frac{E_{b}}{N_{0}},
$$

where $m R$ is the number of information bits sent by a symbol of energy $E_{S}$. Third, good RSC codes for AWGN channels will perform well in BICM-ID systems with overall interleaving.

Figure 4 compares the upper bounds (1) with simulation results of the BICM-ID system for 8-state RSC codes. Bounds have the same behavior as curves of simulation results have, and expected to be good approximation of BER at high SNR.

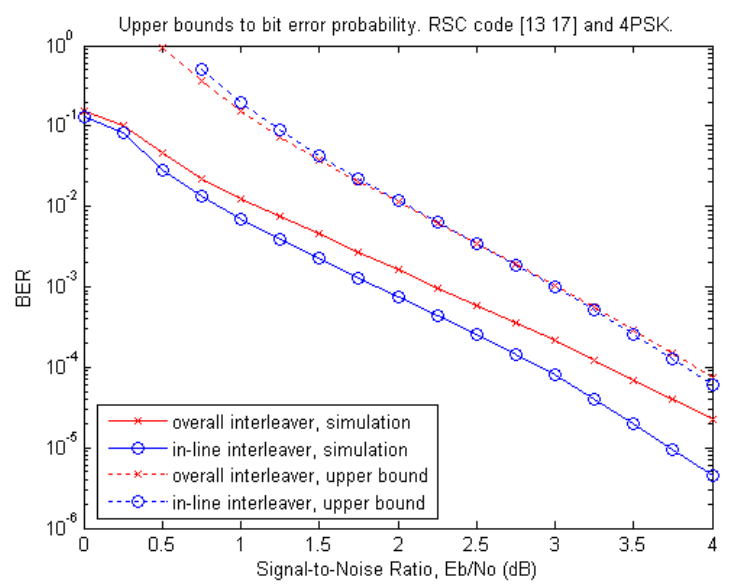

Figure 4. Comparison of upper bounds and simulation results for RSC code $[1,17 / 13]$ with overall and in-line interleaver.

\subsection{The BICM-ID System with In-Line Interleaving}

With in-line interleaving the encoder output bits are not completely decoupled so that each component bit sequence is assigned to a certain binary channel among $m$ parallel channels formed by the $2^{m}$-ary modulation, given IF condition holds.

We consider $2^{m}$-ary modulation and rate- $k / m$ RSC codes. Without loss of generality, we associate systematic bits $c_{t}^{j}$ with the bit distance $d_{E}^{j}, 1 \leq j \leq m$, where $c_{t}=\left(c_{t}^{1}, c_{t}^{2}, \ldots, c_{t}^{m}\right)$ is the output of the RSC encoder at time $t$. For the RSC code, consider an error event of length $L$ associated with binary sequence

$$
C_{L}=c_{1}^{1} c_{1}^{2} \cdots c_{1}^{m}, c_{2}^{1} c_{2}^{2} \cdots c_{2}^{m}, \cdots, c_{L}^{1} c_{L}^{2} \cdots c_{L}^{m} .
$$

It can be verified that

$$
\begin{array}{r}
\sum_{j=1}^{m} W_{H}\left(C_{L}^{j}\right)\left(d_{E}^{j}\right)^{2}=\frac{W_{H}\left(C_{L}\right)}{m} \sum_{j=1}^{m}\left(d_{E}^{j}\right)^{2}+ \\
\frac{1}{m} \sum_{u=1}^{m-1} \sum_{v=u+1}^{m}\left[\left(d_{E}^{u}\right)^{2}-\left(d_{E}^{v}\right)^{2}\right]\left[W_{H}\left(C_{L}^{u}\right)-W_{H}\left(C_{L}^{v}\right)\right],
\end{array}
$$

where $W_{H}\left(C_{L}\right)$ is the Hamming weight of the error sequence and $W_{H}\left(C_{L}^{j}\right)$ is the Hamming weight of the subsequence of the error sequence formed by the bit $c_{t}^{j}$. For example, consider $m=2$ and a rate-1/2 RSC code. Let $C_{L}^{s}$ and $C_{L}^{p}$ be systematic bit and parity check bit subsequences, respectively. We have $W_{H}\left(C_{L}\right)=$ $W_{H}\left(C_{L}^{s}\right)+W_{H}\left(C_{L}^{p}\right)$. The error event sequence has a squared Euclidean distance to the all-zero path

$$
\begin{aligned}
\left(d_{L}\right)^{2}= & W_{H}\left(C_{L}^{s}\right)\left(d_{E}^{1}\right)^{2}+W_{H}\left(C_{L}^{p}\right)\left(d_{E}^{2}\right)^{2} \\
= & \frac{1}{2} W_{H}\left(C_{L}\right)\left[\left(d_{E}^{1}\right)^{2}+\left(d_{E}^{2}\right)^{2}\right]+ \\
& \frac{1}{2}\left[W_{H}\left(C_{L}^{s}\right)-W_{H}\left(C_{L}^{p}\right)\right]\left[\left(d_{E}^{1}\right)^{2}-\left(d_{E}^{2}\right)^{2}\right] .
\end{aligned}
$$

The first term is the average squared Euclidean distance of the same error event when the overall interleaving is used. Interestingly, if the code is matched to the labeling of the signal constellation so that the second term is positive, then there is a gain over the system 
with overall interleaving. In general, it is difficult to optimize the code design according to (4) such that it matches to the signal constellation. We make it simpler by using an upper bound on the error probability.

Remark 3 The equivalent AWGN channel with BPSK modulation for transmission of the $j$-th bit among $m$ RSC encoder output bits has the following SNR

$$
\frac{E_{b}^{j}}{N_{0}^{*}}=\frac{m}{4 R N_{0}}\left(d_{E}^{j}\right)^{2} .
$$

Theorem 2 Consider a BICM-ID system with in-line bit interleaving and transmission of a rate- $k / m$ RSC code over an AWGN channel with one-side noise power spectral density $N_{0}$. Under the assumption of IF and IF-regular labeling, the BEP of the system is upper bounded as follows

$$
P_{b}<\frac{1}{k} \sum_{L=L_{\min }} \sum_{i=1}^{N_{L}} B_{L, i} D^{\sum_{j=1}^{m} W_{H}\left(C_{L, i}^{j}\right)\left(d_{E}^{j}\right)^{2}},
$$

where $N_{L}$ is the number of length-L error events, $B_{L, i}$ is the information weight of the $i$-th length- $L$ error event, and $D=\exp \left\{-m /\left(4 N_{0}\right)\right\}$.

\section{Code Search Results}

Given the signal constellation, we determine its IFregular labeling and carry out the search for each given number of states of the code.

Step 1: Generate a RSC code

Step 2: For each transformation of the selected IFregular labeling by permutation, compute the bound (5) at a given SNR using the technique of [13], with a small modification when the code is punctured. The computed value of (5) is used as the estimate of BER. The encoder configuration and the labeling that give lowest BER estimation at the given SNR are stored for next comparison.

Step 3: The obtained value of (5) is compared with the one stored at the previous round. The lower is stored together with the related combination of the RSC code and labeling.

Step 4: Change the puncturing matrix (if it is in use) or the encoder configuration and repeat steps 1-3 to find the best combination of the RSC encoder and labeling that give lowest value of (5).

We report results of full search for good combination of RSC codes and labelings of signal constellation for the BICM-ID system with in-line interleaving. For the 4point signal constellations (Figure 3) we obtained new rate-1/2 RSC codes in Table I. For the 8PSK constellation we searched for rate- $2 / 3$ RSC codes. We use two approaches in constructing the rate-2/3 RSC codes, namely, with puncturing (Table II) and without puncturing (Table III). Generators of rate-1/2 RSC codes are given in octal and in the form $\left[g_{1}, g_{2}\right]$ or $\left[1, g_{2} / g_{1}\right]$ where $g_{1}$ determines the feedback. Generators of rate-2/3 RSC codes without puncturing are given in the form

$$
\left[\begin{array}{ccc}
g_{11} & 0 & g_{12} \\
0 & g_{21} & g_{22}
\end{array}\right]
$$

\begin{tabular}{|c|c|c|c|c|}
\hline \multirow{2}{*}{$\begin{array}{l}\# \text { of } \\
\text { state }\end{array}$} & \multicolumn{2}{|c|}{ Standard 4PSK } & \multicolumn{2}{|c|}{4 points from $6 \mathrm{PSK}$} \\
\hline & $\begin{array}{c}\text { RSC } \\
\text { encoder }\end{array}$ & Labeling & $\begin{array}{c}\text { RSC } \\
\text { encoder }\end{array}$ & Labeling \\
\hline 4 & {$\left[\begin{array}{ll}7 & 5\end{array}\right]$} & $(0,1,2,3)$ & {$\left[\begin{array}{ll}5 & 7\end{array}\right]$} & $(0,3,4,1)$ \\
\hline 8 & {$\left[\begin{array}{ll}13 & 17\end{array}\right]$} & $(0,1,2,3)$ & {$\left[\begin{array}{ll}17 & 15\end{array}\right]$} & $(0,3,4,1)$ \\
\hline 16 & {$\left[\begin{array}{ll}23 & 35\end{array}\right]$} & $(0,1,2,3)$ & {$\left[\begin{array}{ll}23 & 35\end{array}\right]$} & $(0,4,3,1)$ \\
\hline 32 & {$\left[\begin{array}{ll}73 & 51\end{array}\right]$} & $(0,1,2,3)$ & {$\left[\begin{array}{ll}57 & 65\end{array}\right]$} & $(0,4,3,1)$ \\
\hline 64 & $\begin{array}{|ll|}147 & 135 \\
\end{array}$ & $(0,1,2,3)$ & $\begin{array}{lll}147 & 135 \\
\end{array}$ & $(0,4,3,1)$ \\
\hline 128 & $\left.\begin{array}{ll}373 & 225\end{array}\right]$ & $(0,1,2,3)$ & {$\left[\begin{array}{ll}225 & 373\end{array}\right]$} & $(0,3,4,1)$ \\
\hline 256 & [46 4625$]$ & $(0,1,2,3)$ & | $\left.\begin{array}{lll}677 & 515\end{array}\right]$ & $(0,4,3,1)$ \\
\hline 512 & [1073 1745] & $(0,1,2,3)$ & {$\left[\begin{array}{ll}1073 & 1745\end{array}\right]$} & $(0,4,3,1)$ \\
\hline
\end{tabular}

Table I

BEST RSC ENCODERS FOR 4-POINT CONSTELlations

\begin{tabular}{|c|c|c|c|c|}
\hline \multirow{2}{*}{$\begin{array}{l}\text { \# of } \\
\text { states }\end{array}$} & \multicolumn{2}{|c|}{ SP Labeling } & \multicolumn{2}{|c|}{ SSP Labeling } \\
\hline & $\begin{array}{l}\text { RSC encoder/ } \\
\text { Puncture vector }\end{array}$ & Labeling & $\begin{array}{l}\text { RSC encoder/ } \\
\text { Puncture vector }\end{array}$ & Labeling \\
\hline 4 & $\begin{array}{l}\left(\begin{array}{lll}7 & 5\end{array}\right] \\
\left(\begin{array}{llll}1 & 1 & 1 & 0\end{array}\right)\end{array}$ & $\begin{array}{l}(0,1,2,3, \\
4,5,6,7)\end{array}$ & 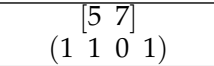 & $\begin{array}{l}(0,2,4,6, \\
5,7,1,3)\end{array}$ \\
\hline 8 & $\begin{array}{c}{\left[\begin{array}{lll}13 & 15\end{array}\right]} \\
\left(\begin{array}{llll}1 & 1 & 1 & 0\end{array}\right) \\
\end{array}$ & $\begin{array}{c}(0,1,2,3, \\
4,5,6,7)\end{array}$ & $\begin{array}{c}{\left[\begin{array}{lll}13 & 15\end{array}\right]} \\
\left(\begin{array}{llll}1 & 1 & 0 & 1\end{array}\right) \\
\end{array}$ & $\begin{array}{l}(0,2,4,6, \\
5,7,1,3)\end{array}$ \\
\hline 16 & $\begin{array}{l}\left(\begin{array}{lll}31 & 23\end{array}\right] \\
\left(\begin{array}{llll}1 & 1 & 0 & 1\end{array}\right)\end{array}$ & $\begin{array}{c}(0,1,2,3, \\
4,5,6,7)\end{array}$ & $\begin{array}{l}\left(\begin{array}{lll}35 & 23\end{array}\right] \\
\left(\begin{array}{llll}1 & 1 & 1 & 0\end{array}\right)\end{array}$ & $\begin{array}{l}(0,2,4,6, \\
5,7,1,3)\end{array}$ \\
\hline 32 & 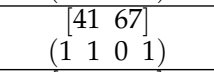 & $\begin{array}{c}(0,1,2,3, \\
4,5,6,7)\end{array}$ & $\begin{array}{c}{\left[\begin{array}{lll}67 & 41\end{array}\right]} \\
\left(\begin{array}{llll}1 & 1 & 1 & 0\end{array}\right)\end{array}$ & $\begin{array}{l}(0,2,4,6, \\
5,7,1,3)\end{array}$ \\
\hline 64 & 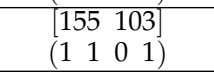 & $\begin{array}{c}(0,1,2,3, \\
4,5,6,7)\end{array}$ & {$\left[\begin{array}{lll}135 & 177\end{array}\right]$} & $\begin{array}{l}(0,2,4,6, \\
5,7,1,3)\end{array}$ \\
\hline 128 & $\begin{array}{l}\left.\mid \begin{array}{llll}343 & 251 \\
(1 & 1 & 0 & 1\end{array}\right) \\
\end{array}$ & $\begin{array}{l}0,1,2,3, \\
4,5,6,7)\end{array}$ & $\begin{array}{l}{\left[\begin{array}{llll}2225 & 307\end{array}\right)} \\
\left(\begin{array}{llll}1 & 1 & 1 & 0\end{array}\right)\end{array}$ & $\begin{array}{l}(0,2,4,6, \\
5,7,1,3)\end{array}$ \\
\hline 256 & 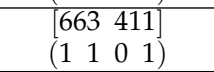 & $\begin{array}{c}(0,1,2,3, \\
4,5,6,7)\end{array}$ & $\begin{array}{l}{\left[\begin{array}{lll}471 & 763\end{array}\right]} \\
\left(\begin{array}{llll}1 & 1 & 1 & 0\end{array}\right)\end{array}$ & $\begin{array}{l}(0,2,4,6, \\
5,7,1,3)\end{array}$ \\
\hline
\end{tabular}

Table II

Best Punctured RSC Encoders for the 8PSK

Table III

Best RSC ENCoders for the 8PSK Constellation.

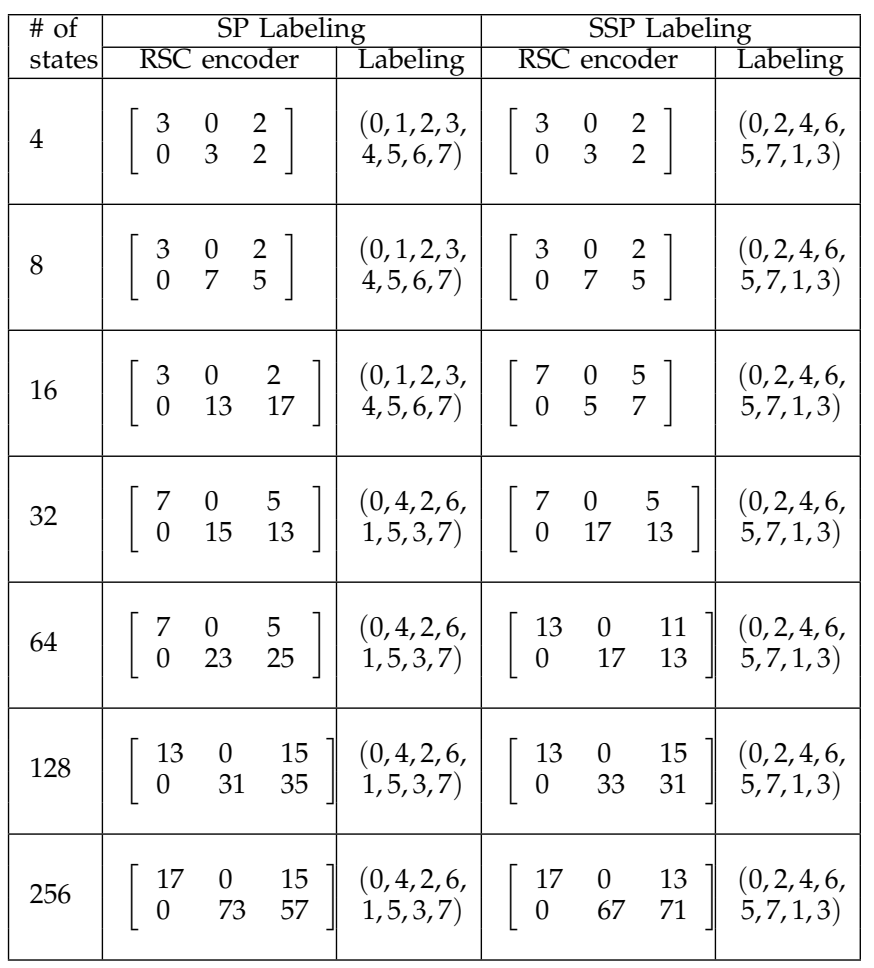




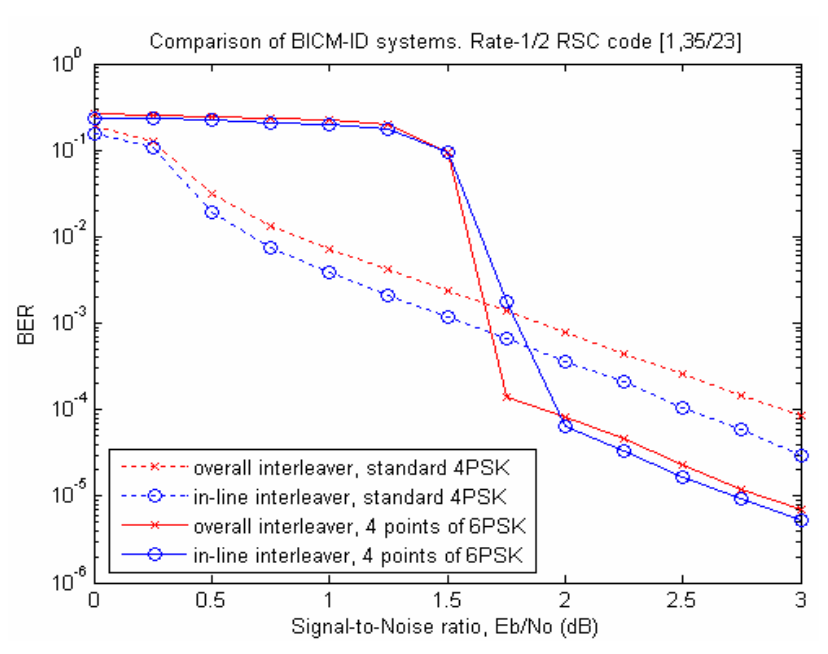

Figure 5. Comparison of BICM-ID systems with overall and in-line interleavers using standard 4PSK and 4 points from 6PSK.

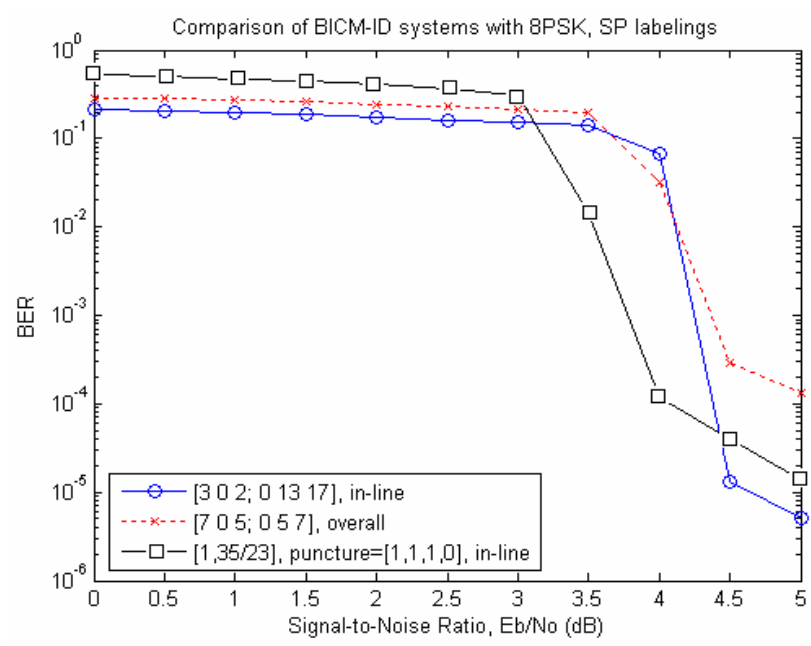

Figure 6. Comparison of BICM-ID systems using the 8PSK constellation with the set partitioning labeling and different 16-state RSC codes.

where $g_{11}$ and $g_{21}$, respectively, determine the feedback for the first and the second systematic bits.

Figure 5 compares the bit error probability of BICMID systems using 16-state RSC code [23 35] and 4point constellations in Figure 3. It can be seen that the 4-point-from-6PSK constellation performs out the standard 4PSK constellation at high SNR. It also can be seen that the in-line interleaver gives rise to a better performance than the overall interleaver does.

Figure 6 compares BICM-ID systems using the 8PSK constellation with the set partitioning labeling and different 16-state rate-2/3 RSC codes. The best code reported so far is the RSC code with generators [7 $05 ; 05$ 7]. It can be seen from the figure that newly found 16-state codes (with and without puncturing) perform out the previously reported code.

\section{Conclusions}

Based on the new concept of BGU labeling of GU signal constellation used in BICM-ID systems, new upper bounds on BEP of the BICM-ID system are reported together with code search results for in-line interleaving and 4PSK, 8PSK signal sets. It seems that the encoder and the labeling must combine to give the best performance of the BICM-ID system with in-line interleaving.

Our simulation results for codes of Tables I-III show that the system with in-line bit interleavers outperform systems with overall bit interleavers, given RSC codes have the same constraint length and information bit frames have the same length.

\section{REFERENCES}

[1] J. Forney, G. D., "Geometrically uniform codes," IEEE Transactions on Information Theory, vol. 37, no. 5, pp. 12411260, Sep. 1991.

[2] A. Calderbank and N. Sloane, "New trellis codes based on lattices and cosets," IEEE Transactions on Information Theory, vol. 33, no. 2, pp. 177-195, Jan. 1987.

[3] S. Benedetto, M. A. Marsan, G. Albertengo, and E. Giachin, "Combined coding and modulation: Theory and applications," IEEE Transactions on Information Theory, vol. 34, no. 2, pp. 223-236, Mar. 1988.

[4] S. Benedetto, R. Garello, M. Mondin, and G. Montorsi, "Geometrically uniform partitions of $l \times$ MPSK constellations and related binary trellis codes," IEEE Transactions on Information Theory, vol. 39, no. 6, pp. 1773-1798, Nov. 1993.

[5] C. Dinh and T. Hashimoto, "A systematic approach to the construction of bandwidth-efficient multidimensional trellis codes," IEEE Transactions on Communications, vol. 48, no. 11, pp. 1808-1817, Nov. 2000.

[6] E. Zehavi, "8-PSK trellis codes for a rayleigh channel," IEEE Transactions on Communications, vol. 40, no. 5, pp. 873-884, May 1992.

[7] X. Li, A. Chindapol, and J. A. Ritcey, "Bit-interleaved coded modulation with iterative decoding and 8 PSK signaling," IEEE Transactions on Communications, vol. 50, no. 8, pp. 1250-1257, Aug. 2002.

[8] X. Li and J. A. Ritcey, "Trellis-coded modulation with bit interleaving and iterative decoding," IEEE Journal on Selected Areas in Communications, vol. 17, no. 4, pp. 715724, Nov. 1999.

[9] R. Garello, G. Montorsi, S. Benedetto, D. Divsalar, and F. Pollara, "Labelings and encoders with the uniform bit error property with applications to serially concatenated trellis codes," IEEE Transactions on Information Theory, vol. 48, no. 1, pp. 123-136, Jan. 2002.

[10] A. Nilsson and T. M. Aulin, "On in-line bit interleaving for serially concatenated systems," in Proc. IEEE Int. Conference on Communications (ICC), vol. 1, Seoul, South Korea, May 2005, pp. 547-552.

[11] A. G. Fàbregas, A. Martinez, and G. Caire, "Bitinterleaved coded modulation," Foundations and Trends in Communications and Information Theory, vol. 5, no. 1-2, pp. 1-153, Jan. 2008.

[12] F. Schreckenbach, P. Henkel, N. Görtz, and G. Bauch, "Analysis and design of mappings for iterative decoding of BICM," in Proc. XI National Symposium on Radio Sciences, Poznan, Germany, 7-8 Apr. 2005.

[13] N. B. Minh and D. T. Cuong, "A tight upper bound on the bit error probability of convolutional codes," in Proc. 8th Vietnam Conference on Radio and Electronics (REV'02), Nov. 2002 


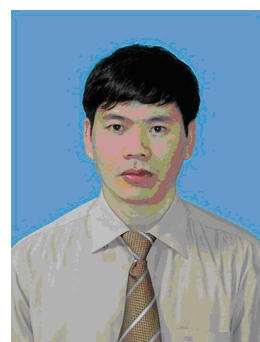

Quoc Khanh Mai received the B.Eng. degree in Radio Electronic Engineering from Le Quy Don Technical University, Hanoi, Vietnam in 1985, the M.Eng. degree in Telecommunication Engineering in Royal Melbourne Institute of Technology (RMIT) - Australia in 1997. $\mathrm{He}$ is currently a senior lecturer at Le Quy Don Technical University, Hanoi, Vietnam. His research interests include coding, networking and electronic measurements.

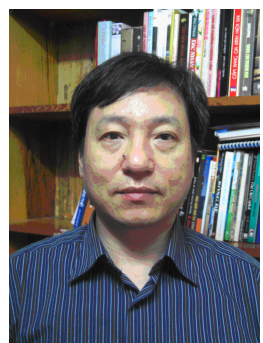

The Cuong Dinh was born in Hanoi, Vietnam, in 1963. He received the B.Es. from Hanoi University of Technology in 1986, the M.Es. and D.E. from the University of ElectroCommunication, Tokyo, Japan, respectively, in 1996 and 1999. He is an Associated Professor in Le Quy Don Technical University, Hanoi, Vietnam. His main interests are on channel coding theory and its application.

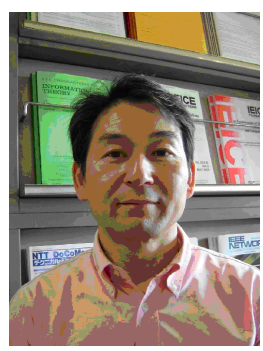

Takeshi Hashimoto was born in Ibaraki Pref., Japan, in 1952. He received the B.Es., M.Es. and D.E. from Osaka University, respectively, in 1975, 1977 and 1981. He was a Research Assistant in Osaka University from 1979 to 1986, was an Associated Professor in Tokyo Denki University from 1986 to 1992, and is a Professor in University of Electro-Communications, Tokyo, Japan. His main interests are on information theory and its application. Dr. Hashimoto is a member of IEEE and SITA. 